safe treatment for men with castration-resistant prostate cancer and result in a durable tumor response. This study also confirms that progression of this malignancy continues to be driven by androgens.

Original article Attard G et al. (2008) Phase I clinical trial of a selective inhibitor of CYP17, abiraterone acetate, confirms that castration-resistant prostate cancer commonly remains hormone driven. J Clin Oncol [doi:10.1200/ JCO.2007.15.9749]

\section{LHRH analog successfully treats infertility in boys with cryptorchidism}

In boys with cryptorchidism, an impaired hormonal surge during early infancy can prevent development of type Ad spermatogonia, which causes infertility. Although the cosmetic appearance of cryptorchidism is successfully treated with early orchiopexy, many patients remain infertile. Luteinizinghormone-releasing hormone $(\mathrm{LHRH})$ analogs increase germ cell numbers, so this study evaluated the effect of the LHRH analog buserelin on fertility in boys with cryptorchidism treated by orchiopexy.

Fifteen boys with unilateral cryptorchidism underwent Schoemaker orchiopexy at a mean age of 3 years. Testicular biopsy revealed an absence of type Ad spermatogonia and fewer than 0.2 germ cells per tubular crosssection. Within 3 months after surgery these boys received buserelin $10 \mu \mathrm{g}$ intranasal spray on alternate days for 6 months. Patients were assessed after hormonal treatment ended, and reassessed at age $>18$ years. Reassessment results were compared with those from 15 age-matched controls who had also undergone Shoemaker orchiopexy (at a mean age of 4 years), but who had not received buserelin.

All adult patients (mean age 19 years) were healthy, with normal sexual development, erectile function and penis length. All patients in the surgery-only group were severely oligospermic (average $1 \times 10^{6}, 95 \% \mathrm{Cl} 0-13 \times 10^{6}$ spermatozoa per ejaculate); by contrast, $86 \%$ of buserelin-treated individuals were normospermic (average $90 \times 10^{6}, 95 \% \mathrm{Cl}$ $53-164 \times 10^{6}$ spermatozoa per ejaculate in the buserelin-treated group; $P=0.000008$ ).

This study demonstrates the successful treatment of cryptorchidism in patients at high risk of infertility. The author suggests that testicular biopsy should be routinely performed at orchiopexy to identify patients without type Ad spermatogonia.

Original article Hadziselimovic F (2008) Successful treatment of unilateral cryptorchid boys risking infertility with LH-RH analogue. Int Braz J Urol 34: 319-328

\section{Adverse outcomes in assisted fertilization might be associated with maternal factors}

Studies have indicated that assisted reproduction is associated with worse perinatal outcomes than spontaneous conception. Romundstad and colleagues, however, noted that poor outcomes could be the result of either assisted reproduction technology or maternal factors. These authors have now established that the outcomes of siblings conceived naturally and those conceived by assisted reproduction do not differ.

The authors first used data from a cohort of 665,883 women included in the Medical Birth Registry of Norway to compare perinatal outcomes of singleton births conceived spontaneously and those conceived by assisted reproduction. Babies conceived via assisted fertilization had reduced birth weight and an increased risk of small size for gestational age (odds ratio [OR] 1.26), premature delivery (OR 1.69), and perinatal mortality (OR 1.31).

A subgroup of 2,546 women had multiple pregnancies, of which at least one each had been conceived naturally and by assisted reproduction. Babies conceived by assisted reproduction did not have a significantly higher risk of premature delivery or of small size for gestational age than those conceived naturally (OR 1.20 and OR 0.99, respectively). Perinatal mortality was higher in spontaneously conceived births than in births conceived by assisted reproduction (OR 0.36).

Romundstad and colleagues suggest that the poor perinatal outcomes reported elsewhere might be attributable to underlying infertility in women who conceive using assisted reproduction technology, rather than the technology itself.

Original article Romundstad LB et al. (2008) Effects of technology or maternal factors on perinatal outcome after assisted fertilisation: a population-based cohort study. Lancet [doi:10.1016/S0140-6736(08)61041-7] 